\title{
A possible link to uracil DNA glycosylase in the synergistic action of HDAC inhibitors and thymidylate synthase inhibitors
}

\author{
Meredith S. Showler ${ }^{1}$ and Brian P. Weiser ${ }^{2^{*}}$ (i)
}

\begin{abstract}
It is well established that thymidylate synthase inhibitors can cause cellular toxicity through uracil DNA glycosylase (UNG2)-dependent pathways. Additionally, thymidylate synthase inhibitors and HDAC inhibitors are known to act synergistically in a variety of cancer types. A recent article from J. Transl. Med. links these together by demonstrating widespread depletion of UNG2 levels across a variety of cell lines treated with HDAC inhibitors. Recent findings suggest that UNG2 depletion by HDAC inhibitors would likely be an effective method to sensitize cells to thymidylate synthase inhibitors. This is particularly important for cancer types that are typically resistant to thymidylate synthase inhibitors, such as cells that are deficient in p53 activity.
\end{abstract}

Keywords: Hdaci, Histone deacetylase inhibitor, Thymidylate synthase inhibitor, UNG2, Uracil DNA glycosylase, p53, 5-Fluorouracil

\section{Main text}

In a recent article from J. Transl. Med., Iveland et al. showed that HDAC inhibitors caused a comprehensive and widespread depletion of Uracil DNA Glycosylase (UNG2) protein levels in a variety of cancer cell lines [1]. This work has implications for cancer treatments that can be affected by UNG2 activity, namely thymidylate synthase (TS) inhibitors, which include 5-fluorouracil, pemetrexed, and raltitrexed. It is well known that TS inhibitors produce toxicity through UNG2-dependent mechanisms in only certain cancer types [2-5]. We propose that Iveland et al. may have uncovered a novel approach to re-sensitize other cancers to TS inhibitors by targeting UNG2-dependent pathways with HDAC inhibitors.

Since the early 2000s, HDAC inhibitors have been approved for clinical use as cancer treatments, and new

\footnotetext{
*Correspondence: weiser@rowan.edu

2 Department of Molecular Biology, Rowan University School of Osteopathic Medicine, Stratford, NJ 08084, USA

Full list of author information is available at the end of the article
}

generations of molecules continue to be developed. These follow the weak HDAC inhibitor valproic acid, which has been used for decades to treat neurological disorders. Classically, HDAC inhibitors are thought to alter gene transcription by modifying the acetylation state of lysine residues on histone tails, thereby altering chromatin structure and transcription factor access. Transcriptional changes presumably alter the levels of critical proteins that affect cell viability or proliferation [1]. Alternatively, HDACs are known to have many non-histone protein substrates whose acetylation state is also enhanced by HDAC inhibitors, and the acetylation of these proteins can affect their function and stability [6]. Thus, uncovering changes to the proteome and protein post-translational modifications after HDAC inhibitor treatments is an important step in understanding their mechanisms of action and refining their clinical utility.

Interestingly, Iveland et al. found in their proteomic work that treating cancer cells with HDAC inhibitors universally reduced protein levels of UNG2, which is the primary enzyme responsible for removing uracil bases

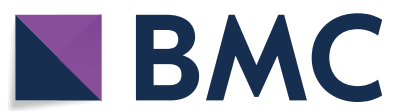

(c) The Author(s) 2020. This article is licensed under a Creative Commons Attribution 4.0 International License, which permits use, sharing, adaptation, distribution and reproduction in any medium or format, as long as you give appropriate credit to the original author(s) and the source, provide a link to the Creative Commons licence, and indicate if changes were made. The images or other third party material in this article are included in the article's Creative Commons licence, unless indicated otherwise in a credit line to the material. If material is not included in the article's Creative Commons licence and your intended use is not permitted by statutory regulation or exceeds the permitted use, you will need to obtain permission directly from the copyright holder. To view a copy of this licence, visit http://creativeco mmons.org/licenses/by/4.0/. The Creative Commons Public Domain Dedication waiver (http://creativecommons.org/publicdomain/ zero/1.0/) applies to the data made available in this article, unless otherwise stated in a credit line to the data. 
from genomic DNA [1, 7]. The comprehensive depletion of UNG2 was observed in eight human cell lines and was consistent with the reduced UNG2 levels that were reported by others examining HDAC inhibitors in select cell types [8-10]. The reduction of UNG2 was impressively rapid after treatment with a chemically diverse set of molecules (SAHA, MS275, valproate, and sodium butyrate). The reason for reduced UNG2 levels is that HDAC inhibitors induce hyperacetylation of UNG2 [1], which can facilitate its interaction with a ubiquitin ligase [10], thereby resulting in UNG2's degradation by the proteasome.

Cellular UNG2 levels are important for the toxicity of TS inhibitors, which elevate genomic uracil content by depleting dTTP pools in favor of dUTP [2]. Polymerases then utilize dUTP during replication, which puts the stability of the genome at risk [3, 4, 11]. UNG2 initiates base excision repair pathways to remove genomic uracil and restore DNA to its original code. Therefore, UNG2 activity often mitigates toxicity associated with TS inhibitors in the context of genomic uracilation.

However, the role of UNG2 in TS inhibitor treatments has historically been complex. The toxicity of TS inhibitors in some cell types is entirely independent of UNG2 activity [2,5]. Recent work has provided a basis for this by demonstrating a strong link between TS inhibitors, UNG2 levels, and p53 activity [5]. Functional wildtype p53 activity is typically associated with a sensitive response of cancer cells to TS inhibitors regardless of UNG2 activity $[5,12]$. This is because p53 is important for the activation of apoptosis in cells treated with TS inhibitors [5]. In contrast, cells that are deficient in p53 or harbor mutations that compromise p53 activity are generally resistant to TS inhibitors [5, 12]. However, this resistance can be overcome by depleting UNG2, which elevates genomic uracil content and DNA instability, and promotes apoptosis through p53-independent pathways [3-5].

Interestingly, cells that lack wild-type p53 are typically resistant to TS inhibitors, but can be sensitized to their toxicity by co-treatment with HDAC inhibitors [12]. In fact, HDAC inhibitors and TS inhibitors are well known to behave synergistically against a diverse panel of cancer types [9, 12-14]. This has been attributed to the ability of HDAC inhibitors to also reduce TS expression at the gene and protein level $[1,9,12,13]$, which allows lower doses of TS inhibitor to be efficacious. Indeed, Iveland et al. also measured reduced TS protein levels in cells treated with HDAC inhibitors. It is quite notable that HDAC inhibitors can reduce protein levels of both TS and UNG2, which are critical components of a common pathway governing pyrimidine metabolism and DNA repair $[1,2]$, and that reduction of TS and UNG2 occur through both transcriptional and post-translational mechanisms [1,9]. We note that HDAC inhibitors can also impact the expression of additional proteins involved in pyrimidine metabolism, such as thymidine phosphorylase, which regulates the efficacy of other fluoropyrimidine anti-metabolites [15].

We propose that the synergistic action of HDAC inhibitors and TS inhibitors in cells lacking p53 activity is facilitated not only by the downregulation of TS, but also by the reduction of UNG2 levels caused by HDAC inhibitors [12]. Indeed, genetic depletion of UNG2 sensitizes p53 deficient cells to TS inhibitors in a manner that resembles the sensitization caused by HDAC inhibitors [5]. In both cases, a p53-independent apoptosis pathway that is not normally triggered by TS inhibitors becomes activated [5, 12].

The newfound ability to disrupt uracil base excision repair with HDAC inhibitors has immense potential for cancer treatment. UNG2 levels have been pharmacologically reduced with HDAC inhibitors in diverse cell lines that include HT29, HeLa, HEK293, Jurkat, SUDHL5, DLD1, HaCaT, A549, HCT116, and HAP1 [1, 9, 10]. We note that several of these cell lines also lack normal p53 activity and are resistant to TS inhibitors unless they are sensitized pharmacologically or genetically (e.g., HT29 cells which harbor mutant p53) $[5,9]$. Providing further pharmacologic specificity is the fact that the selective HDAC inhibitor MS275, which targets HDACs 1-3 [16], reduced UNG2 protein to similar levels as non-selective pan-HDAC inhibitors [1]. This presents a clear path for co-targeting specific HDAC proteins and TS in p53 deficient cells, and evaluating toxicity associated with deficient uracil base excision repair.

The experimental work we discussed could lead to more effective clinical treatments for cancer types that show resistance to TS inhibitors. However, several challenges have been presented thus far during early clinical trials using HDAC inhibitor/TS inhibitor combination therapies of SAHA and 5-fluorouracil [17-19]. Most notably, the well-described ability of SAHA to reduce TS expression in cells has not consistently occurred in solid tumors $[1,9,12,13]$, which hinders proposed mechanisms of SAHA/5-fluorouracil synergy [17-19]. In these clinical trials, it was likely that the SAHA concentrations sustained over time in the tumor were insufficient to reduce TS protein levels, which would not arise as an issue during tissue culture experiments [1]. The ability of SAHA to reduce UNG2 levels in solid tumors has also not been examined. The challenges of targeting these proteins in solid tumors may be overcome with different HDAC inhibitors with improved pharmacologic profiles and/or optimized dosing regimens. This should be combined with biomarker technologies that examine p53 
status and measure levels of TS and UNG2 before and after drug treatments. Together, these findings should enhance the promising strategy of targeting pyrimidine metabolism pathways in cancers with diverse p53 status and varying sensitivity to TS inhibitors.

\author{
Abbreviations \\ HDAC: Histone deacetylase; TS: Thymidylate synthase; UNG2: Uracil DNA \\ glycosylase.
}

\section{Acknowledgements}

Not applicable.

\section{Authors' contributions}

MSS and BPW wrote the manuscript. Both authors read and approved the final manuscript.

\section{Funding}

Not applicable.

\section{Availability of data and materials}

Not applicable.

\section{Ethics approval and consent to participate}

Not applicable.

\section{Consent for publication}

Not applicable.

\section{Competing interests}

The authors declare that they have no competing interests.

\section{Author details}

${ }^{1}$ Biology Department, Wheaton College, Wheaton, IL 60187, USA. ${ }^{2}$ Department of Molecular Biology, Rowan University School of Osteopathic Medicine, Stratford, NJ 08084, USA.

Received: 12 June 2020 Accepted: 27 September 2020

Published online: 07 October 2020

\section{References}

1. Iveland TS, Hagen L, Sharma A, Sousa MML, Sarno A, Wollen KL, et al. HDACi mediate UNG2 depletion, dysregulated genomic uracil and altered expression of oncoproteins and tumor suppressors in B- and T-cell lines. J Transl Med. 2020;18:159.

2. Grogan BC, Parker JB, Guminski AF, Stivers JT. Effect of the thymidylate synthase inhibitors on dUTP and TTP pool levels and the activities of DNA repair glycosylases on uracil and 5-fluorouracil in DNA. Biochemistry. 2011;50:618-27.

3. Bulgar AD, Weeks LD, Miao Y, Yang S, Xu Y, Guo C, et al. Removal of uracil by uracil DNA glycosylase limits pemetrexed cytotoxicity: overriding the limit with methoxyamine to inhibit base excision repair. Cell Death Dis. 2012;3:e252.

4. Weeks LD, Zentner GE, Scacheri PC, Gerson SL. Uracil DNA glycosylase (UNG) loss enhances DNA double strand break formation in human cancer cells exposed to pemetrexed. Cell Death Dis. 2014;5:e1045.
5. Yan Y, Qing Y, Pink JJ, Gerson SL. Loss of uracil DNA glycosylase selectively resensitizes p53-mutant and-deficient cells to 5-FdU. Mol Cancer Res. 2018;16:212-21.

6. Singh BN, Zhang G, Hwa YL, Li J, Dowdy SC, Jiang S-W. Nonhistone protein acetylation as cancer therapy targets. Expert Rev Anticancer Ther. 2010;10:935-54.

7. Kavli B, Sundheim O, Akbari M, Otterlei M, Nilsen H, Skorpen F, et al. hUNG2 is the major repair enzyme for removal of uracil from $U: A$ matches, U: G mismatches, and U in single-stranded DNA, with hSMUG1 as a broad specificity backup. J Biol Chem. 2002;277:39926-36.

8. Chai G, Li L, Zhou W, Wu L, Zhao Y, Wang D, et al. HDAC inhibitors act with 5-aza-2'-deoxycytidine to inhibit cell proliferation by suppressing removal of incorporated abases in lung cancer cells. PLOS ONE. 2008;3:e2445.

9. LaBonte MJ, Wilson PM, Fazzone W, Groshen S, Lenz H-J, Ladner RD. DNA microarray profiling of genes differentially regulated by the histone deacetylase inhibitors vorinostat and LBH589 in colon cancer cell lines. BMC Med Genomics. 2009;2:67.

10. Bao Y, Tong L, Song B, Liu G, Zhu Q, Lu X, et al. UNG2 deacetylation confers cancer cell resistance to hydrogen peroxide-induced cytotoxicity. Free Radic Biol Med. 2020;160:403-17.

11. Huehls AM, Huntoon CJ, Joshi PM, Baehr CA, Wagner JM, Wang X, et al. Genomically incorporated 5-fluorouracil that escapes UNG-initiated base excision repair blocks DNA replication and activates homologous recombination. Mol Pharmacol. 2016;89:53-62.

12. Gennaro ED, Bruzzese F, Pepe S, Leone A, Delrio P, Subbarayan P, et al. Modulation of thymidilate synthase and p53 expression by HDAC inhibitor vorinostat resulted in synergistic antitumor effect in combination with 5FU or raltitrexed. Cancer Biol Ther. 2009:8:782-91.

13. Lee J-H, Park J-H, Jung Y, Kim J-H, Jong H-S, Kim T-Y, et al. Histone deacetylase inhibitor enhances 5 -fluorouracil cytotoxicity by downregulating thymidylate synthase in human cancer cells. Mol Cancer Ther. 2006:5:3085-95.

14. Garg D, Henrich S, Salo-Ahen OMH, Myllykallio H, Costi MP, Wade RC. Novel approaches for targeting thymidylate synthase to overcome the resistance and toxicity of anticancer drugs. J Med Chem. 2010:53:6539-49.

15. Di Gennaro E, Piro G, Chianese MI, Franco R, Cintio AD, Moccia T, et al. Vorinostat synergises with capecitabine through upregulation of thymidine phosphorylase. Br J Cancer. 2010;103:1680-91.

16. Gryder BE, Wu L, Woldemichael GM, Pomella S, Quinn TR, Park PMC, et al. Chemical genomics reveals histone deacetylases are required for core regulatory transcription. Nat Commun. 2019;10:3004.

17. Wilson PM, El-Khoueiry A, lqbal S, Fazzone W, LaBonte MJ, Groshen S, et al. A phase I/II trial of vorinostat in combination with 5 -fluorouracil in patients with metastatic colorectal cancer who previously failed 5-FUbased chemotherapy. Cancer Chemother Pharmacol. 2010;65:979-88.

18. Fakih MG, Pendyala L, Fetterly G, Toth K, Zwiebel JA, Espinoza-Delgado I, et al. A phase I, pharmacokinetic and pharmacodynamic study on vorinostat in combination with 5-fluorouracil, leucovorin, and oxaliplatin in patients with refractory colorectal cancer. Clin Cancer Res. 2009;15:3189-95.

19. Fakih MG, Groman A, McMahon J, Wilding G, Muindi JR. A randomized phase II study of two doses of vorinostat in combination with 5-FU/LV in patients with refractory colorectal cancer. Cancer Chemother Pharmacol. 2012;69:743-51.

\section{Publisher's Note}

Springer Nature remains neutral with regard to jurisdictional claims in published maps and institutional affiliations. 